\title{
Electrical conductive polyaniline in various nanostructures for corrosion inhibition of carbon steel
}

\author{
Ahmed A. Al-Dulaimi *1, S. Hashim², Lway Faisal Abdulrazak ${ }^{3}$, M. Husham ${ }^{4}$ \\ ${ }^{1}$ MICET, Universiti Kuala Lumpur, Lot 1988, Taboh Naning 78000 Alor Gajah, Melaka, \\ Malaysia. \\ *Email: ahmedulaimi@unikl.edu.my \\ ${ }^{2}$ Department of Bioprocess and Polymer Engineering, Faculty of Chemical and energy \\ Engineering, UTM skudai, Malaysia \\ ${ }^{3}$ Cihan research center, Computer science department, Cihan University-Sulaimaniyah, \\ Iraq. \\ ${ }^{4}$ Institute of Advanced Technology (ITMA), Universiti Putra Malaysia, 43400 Serdang, \\ Kuala lumpur, Malaysia.
}

\begin{abstract}
Conductive polyaniline (PANI) in various nanostructures were prepared by situ polymerization for corrosion protection application of carbon steel. Anticorrosion performance of the samples was evaluated by applying salt spray and adhesion tests based on standards ASTM B117 and ASTM D3359 respectively. Synthesis of PANI in various nanostructures carried out by doping aniline monomer with phosphoric acid in four different molar ratios. Field emission scanning electron microscopy (FESEM) images confirms the variation in nanostructures of samples. The samples were also characterized using Fourier transform infrared (FTIR), X-ray diffraction (XRD), electrical conductivity and dispersion stability in ethanol medium. The transition of PANI nanostructure from nanofibers to nanorods and nanospheres occurred with the increase of molar ratios (phosphoric acid/aniline) from $0.1,0.5,1$ and 2 respectively. The results show that coating containing PANI nanofibers has the best resistance against corrosive aggression due to the high dispersion stability and decent uniformity. Accordingly, this work finds that the morphological structure and dispersion stability for PANI has a significant impact on the anticorrosion performance.
\end{abstract}

Keywords: Polyaniline; nanostructures, micelle; corrosion protection.

\section{INTRODUCTION}

Corrosion caused severe damage of structures such as aircrafts, automobiles, buildings, bridges and gas pipelines. Corrosion is a various interaction between the iron and the atmosphere causing a degradation of the iron properties. Interaction with oxygen lead to the formation of iron oxide layers on the metal surface. In a moisten environment, corrosion occurred because of electrochemical processes of metal ion transport and reaction. The most common techniques for corrosion prevention is painting metals surface with anticorrosive coatings. Usually, coatings applied in corrosion inhibition are thermosetting resins, such as polyurethane, acrylic, epoxy, etc. [1-3]. Conductive polymers attract numerous interest at the last decades due to their environmentally friendly behavior and wide range of applications especially their unique corrosion inhibiting for metals. Polyaniline (PANI) is most promising 
conductive polymer due to its usefulness in wide range of applications and interesting redox properties associated with the chain of nitrogen [4,5].

Recent reports showed that PANI possess a decent chemical and environmental stability and protecting metals against corrosion attack via passivation of oxide layer shell occurs between metal and coating [6,7]. Moreover, researches proved that dispersed PANI in the polymeric matrix coating on the steel can passivate the pinholes and the defects of metal[8,9]. PANI in nanoscales dimensions possess high potential due to the unique reactivity in high surface area. They have used to enhance performance of several applications such as anticorrosion and antistatic coatings, energy storages, electronic devices, capacitors, biosensors, etc. [10,11]. However, PANI prepared in several nanostructures forms such as nanotube[12], nanospheres, nanowires [13] and nanofibers [14]each form shows different physical and chemical properties[15].

Self-assembling mechanism in micelle method is successfully applied to prepare PANI in different nanostructures. The morphological properties determine based on ratios between aniline (monomer) and acid (dopant) during polymerization process[16,17]. Xia and his group reported about controlling the arrangements of PANI in nanorods form using different molar ratios between aniline and Allura Red AC (director). Results concluded that PANI morphology highly depends on the ratios of monomer to director [18]. Recently, one dimension (1D) nanostructures PANI such as nanotubes, nanofibers, nanowires, and nanorods captured wide interest due to the high potential in many application fields that demands low dimensions and high surface area [19]. The template free "self-assembly" method have been applied as a facile procedure to prepare PANI in 1D nanostructures such as (nanorods, nanofibers and nanotubes). Self-assembly procedure employ the aniline salts (anilinium) arrangement (acids molecules-aniline) attraction into a nanostructured intermediate arrangement before the polymerization initiated [20]. However, anilinium structure can be control by adjusting the ratios between acid to aniline. The transitions of PANI nanostructures from nanoflakes to nanorods then to nanospheres was obtained with increasing the molar ratios of acid to aniline monomer [21]. The observation of Polymer in different nanostructures have been associated with the changes of reaction parameters that results variation in molecular structures. [22].

In this work, we prepared PANI in 1D nanostructures using template free "selfassembly" method for corrosion inhibition employments. Phosphoric acid is a promising candidate for this doping system with high capacity to protect metal against corrosion atmospheres $[23,24]$. The investigates of anticorrosion performance for PANI in various nanostructures, have not been addressed yet. The morphological properties were tested by field emission scanning electron microscopy (FESEM) and anticorrosion performance studied by salt spray with adhesion tests based on standards ASTM B117 and ASTM D3359 respectively. Electrical conductivity and dispersion stability was detected for samples. XRD diffraction used to exam crystallinity of samples and functional groups investigated by FTIR spectra.

\section{METHODS AND MATERIALS}

\section{Synthesis of PANI Nanostructures}

The aniline monomer purchased from Mallinckrodt U.S.A. The phosphoric acid (dopant) was provided by MERCK Germany. The initiator of radical polymerization ammonium persulfate (APS) was provided by QRëC Germany. Nippon Paint Malaysia is a source of acrylic matrix, testing salt $\mathrm{NaCl}$ from (MERCK Germany) and the plates of carbon steel were provided from local factories. Initially, four samples of 0.2 mole of aniline monomer were dissolved in 200 
$\mathrm{mL}$ de-ionized water doped with four different phosphoric acid concentrations $(0.02,0.1,0.2$ and 0.4 moles). The mixtures were stirred for about 10 minutes using ultrasonic stirrer. Precooled $200 \mathrm{ml}$ of 0.2 mole water solution of APS reaction initiator was added to the mixtures with constant stirring at $5 \pm 1{ }^{\circ} \mathrm{C}$ in nitrogen atmosphere. The mixtures were then allowed to stir up to 8 hours to confirm the reaction completion. The suspensions in dark green color which is indicate to form PANI (emeraldine salt). The suspensions were then collected in a funnel buchner then washed properly by distilled water. The collected samples then kept in vacuum oven for one day to dry at moderate temperature. Lastly, the dried samples then exposed to grind using grinding machine prior to apply in coating system.

\section{Preparation and Characterization}

The prepared samples were examined chemically using Perkin Elmer model of Fouriertransform infrared spectroscopy at spectrum range $4000-400 \mathrm{~cm}^{-1}$. XRD analysis of Siemens D5000 Diffractometer used to study samples crystallinity. Samples in powder form compressed in a pellets to measure electrical conductivity at room temperature using the digital Multimeter model CP7676 Actron. Morphological diversity was studyed by electron microscopy (FESEM) model JEOL JSM6701F. Three PANI samples (nanofibers, nanorods and nanospheres) were blended with acrylic paint using laboratory attritor to prepare the coatings. In order to avoid gel formation and solvent evaporation, the coatings were kept in airtight jar [25]. Corrosion prefrmance of coated carbon steel samples carried out by salt spray chamber brand SF/450/CCT. Carbon steel samples were cut eith dimensions of length 12.7, width $7.6 \mathrm{~cm}$ and $0.8 \mathrm{~cm}$ thickness according to ASTM B117 standard. The samples were polished by fine size emery paper (600 grit), cleaned, degreased with industrial grade acetone. Sample shape and size was made convenient to laboratory corrosion tests according to ASTM B117 standard. After coatings completely dry, slots were made in the coatings with considering depth and length. Corrosion evaluated by visual monitoring and adhesion strength testing according to ASTM D3359 standard after 35 days of salt spray exposion period [8].

\section{RESULTS AND DISCUSSIONS}

Four doped aniline monomers with phosphoric acid were synthesized by in-situ polymerization using the oxidizing agent ammonium persulfate as a radical initiator. Four molar ratios of (phosphoric acid/aniline) solutions $(0.1,0.5,1$ and 2) with fixing molarities ratio for oxidant agent ammonium persulfate at 0.2 mole. Figure 1 shows the transition in PANI nanostructures accompanied with the increase (Phosphoric acid/Aniline) ratios. When the ratios were 0.1 and 0.5 (Figure $1 \mathrm{a}, \mathrm{b}$ ), PANI structures tend to assemble in cylinder forms (fibers and rods) respectively in Table 1.

Table 1. influence of the acid/monomer rations on the PANI morphology

\begin{tabular}{ccccc}
\hline $\begin{array}{c}\text { Dopant } \mathrm{H}_{3} \mathrm{PO}_{4} \\
(\mathrm{~mole})\end{array}$ & $\begin{array}{c}\text { Monomer } \\
\text { Aniline (mole) }\end{array}$ & $\begin{array}{c}\text { Dopant / } \\
\text { monomer (ratios) }\end{array}$ & Nanostructures & $\begin{array}{c}\text { Average } \\
\text { Diameter }(\mathrm{nm})\end{array}$ \\
\hline 0.02 & 0.2 & 0.1 & Nanofibers & $25-50$ \\
0.1 & 0.2 & 0.5 & Nanoroads & $155-165$ \\
0.4 & 0.2 & 2 & Nanospheres & $25-33$ \\
\hline
\end{tabular}

While the structures of PANI formed in spherical shape at 2.0 molar ratios (Figure1 d). Besides, at equal ratio, between monomer and acid dopant encourage the PANI to forma mixtures of nonspecific form of fibers and spherical particles (Figure1 c). The variation in the PANI morphology probably due to micelle aggregation and arrangements [26]. However, 
PANI morphologies control through use of special surfactant to pre-arrange aniline monomers prior to the synthesis step. Sodium dodecyl benzene sulfate (SDBS) which is contributing mainly as surfactant to formulate micelle like nanotube prior/during the polymerization process [27]. Another valuable mechanism applied to control the configuration of aniline monomer and manipulate PANI nanostructures with the self-assembly arrangement. Anilinium salt was formed through (acid-based) reaction between phosphoric acid and aniline monomer.

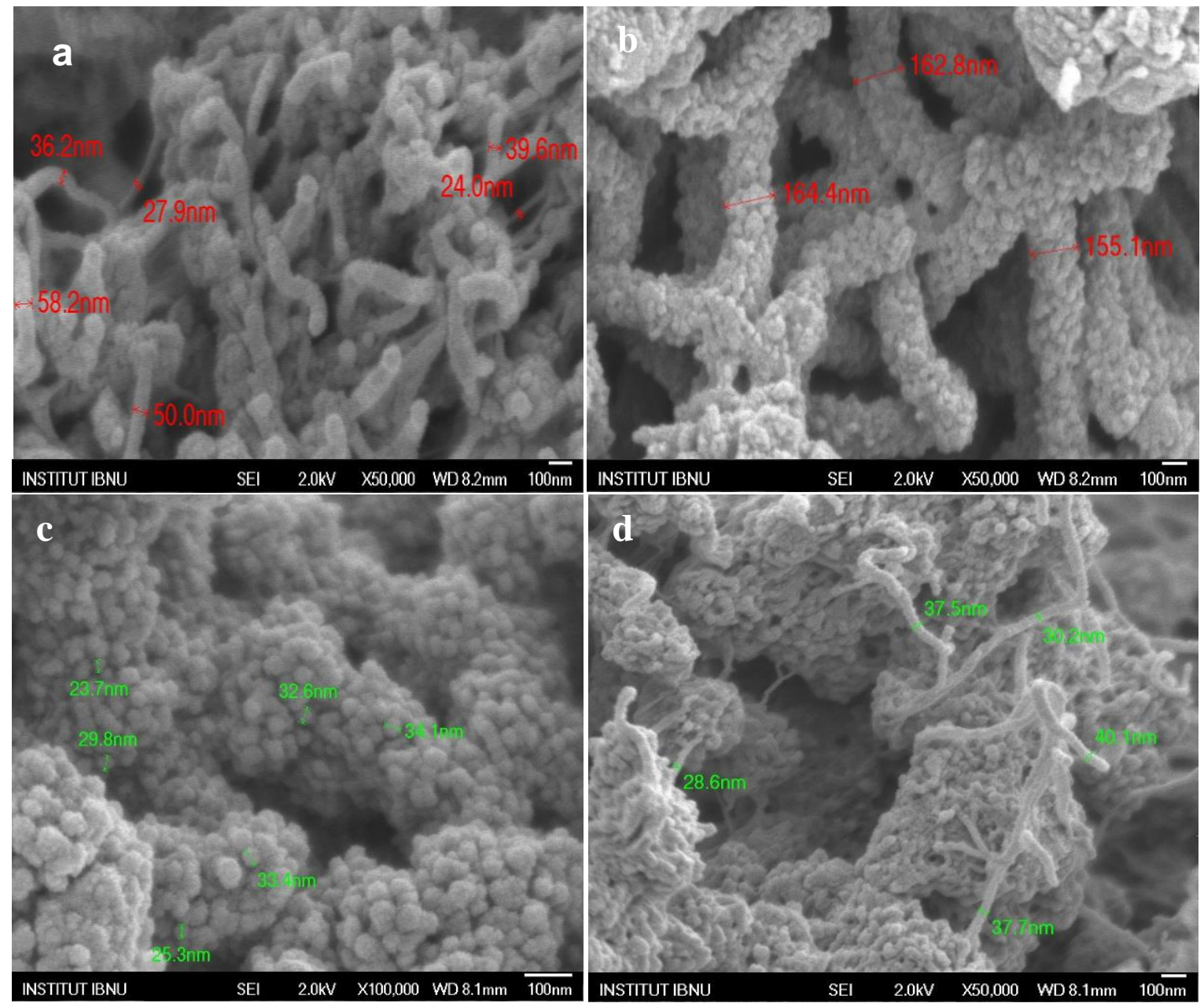

Figure 1. FESEM images shows transtion morphologies of PANI nanostructures (a) nanofibers, (b) nanoroads, (c) nanospheres and (d) nanofibers and nanspheres mix.

Figure 2 designates the anilinium salt interactions of (dopant-monomer) represented by zigzag tail and the free synthesized aniline represented by oval head. Anilinium salt (dopant-monomer) can act as a template to form micelle arrangements. However, the phenyl ring in aniline monomer is a (hydrophobic) terminal while $\mathrm{H}_{3} \mathrm{PO}_{4}$ act as (hydrophilic), this product will enhance formation of micelle arrangement. When the morality of acid dopants was less than monomers, means a few amounts of anilinium salts were consisted in the reaction medium and a little existence of template with more free aniline. The free aniline diffused stimulates the elongation condition to micelles cylinder-like. After adding initiator; the free aniline was synthesized sequentially on the surface of micelles cylinder-like to produce PANI nanorods and nanofibers. The diameter of cylinders forms turns to decrease with decreases of these acid-monomer ratios[14]. However, increasing acid morality lead to increase existent of anilinium salts template with little free aniline was diffused. Micelles sphere-like was existed and resulted PANI nanospheres after injecting the oxidant agent. 

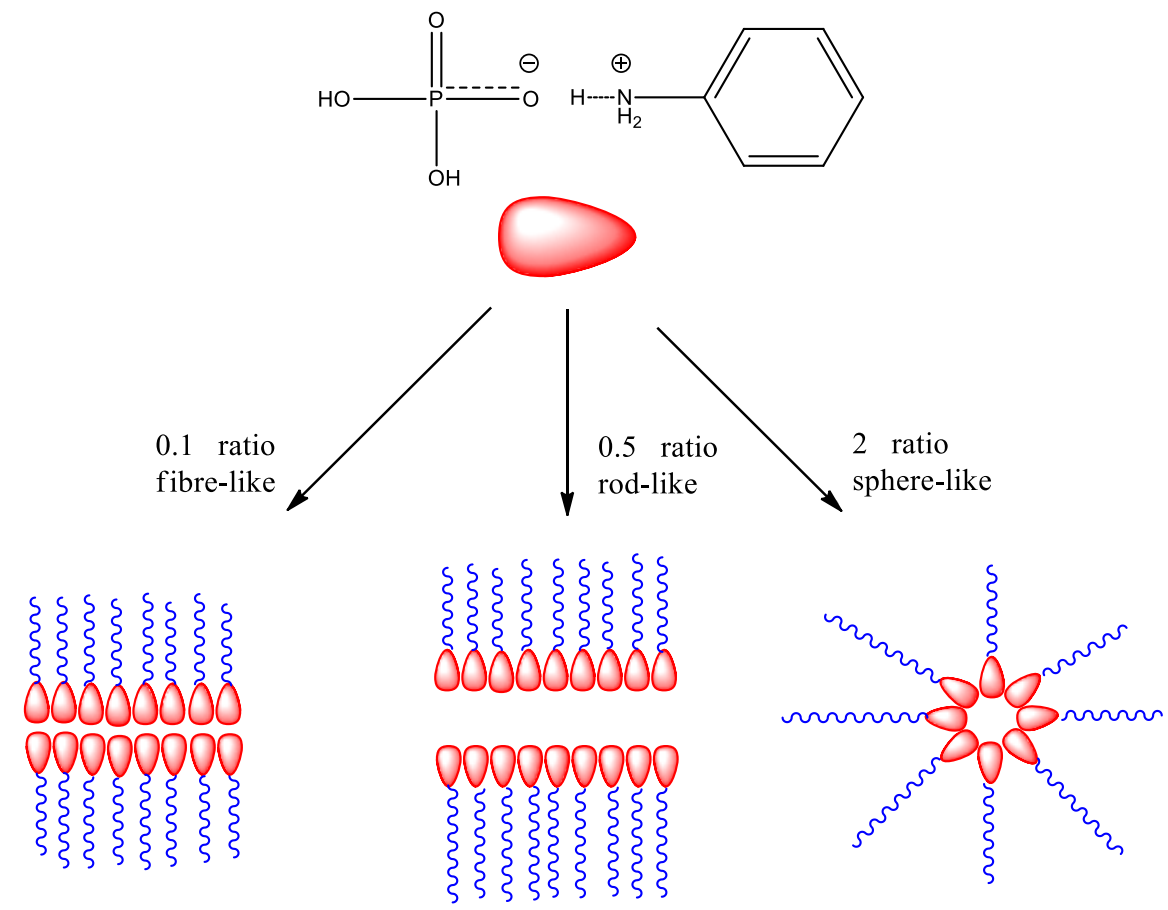

Fiure 2. Self-assembling mechanism in micelle method for anilinium salt.

FTIR results revealed that PANI spectra were similar (Figure 3) to PANI doped with organic [28] and inorganic acids [20]. The bands around $1600 \mathrm{~cm}-1$ and $1500 \mathrm{~cm}^{-1}$ are indicated to the stretching bond of $\mathrm{C}=\mathrm{N}$ and $\mathrm{C}=\mathrm{C}$ bonds [29]. The bands about $1250 \mathrm{~cm}^{-1}$ and $1300 \mathrm{~cm}^{-1}$ are referred to the stretching of benzenoid ring in $\mathrm{C}-\mathrm{N}$ and $\mathrm{C}-\mathrm{N}-\mathrm{C}$ bonds respectively [30]. The band at $1117 \mathrm{~cm}^{-1}$ is assigned to a plane bending vibration of $\mathrm{C}-\mathrm{H}$ mode which is found during protonation. The band which is falls in the range of $800 \mathrm{~cm}-1$ refers to 1, 4-di-substituted benzene [5].

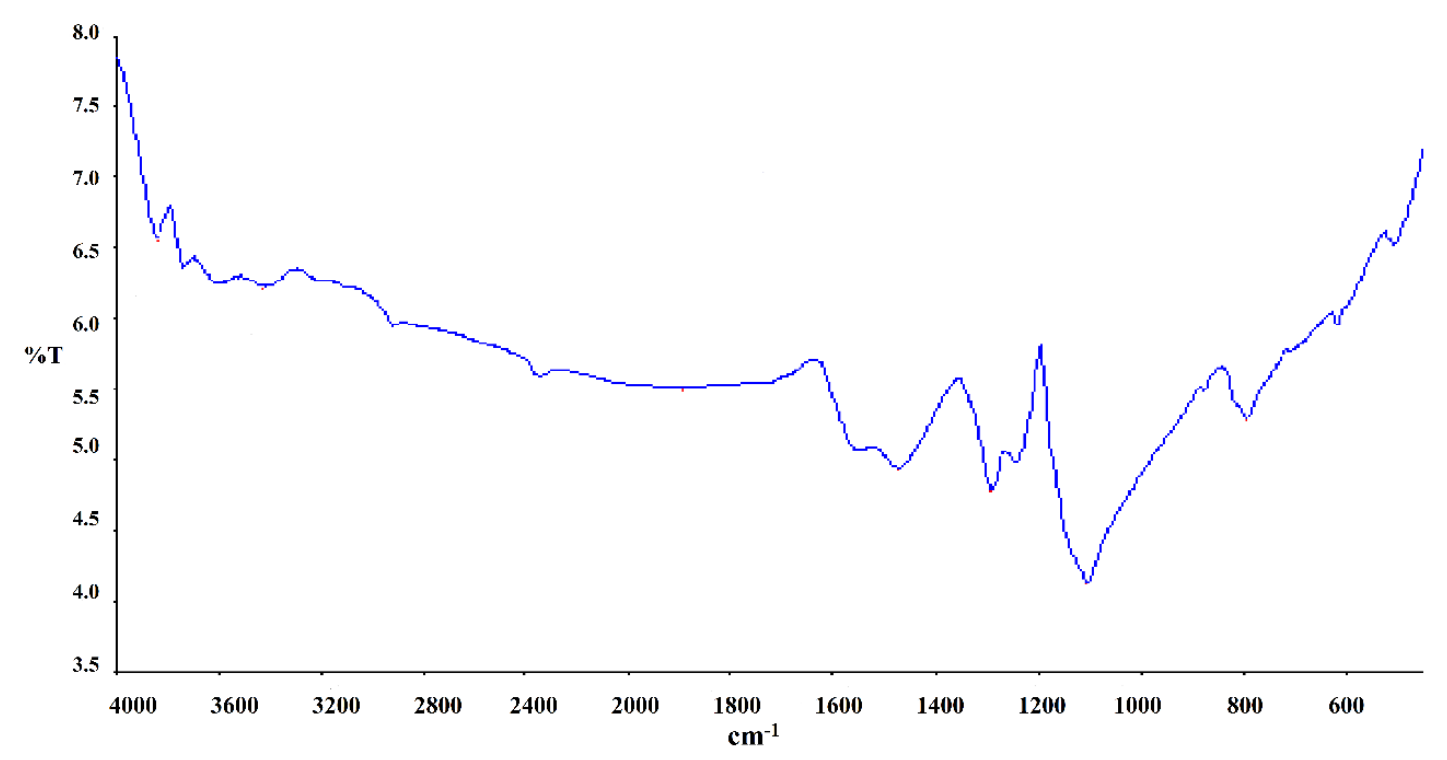

Fiure 3. FTIR spectra of PANI in various nanostructures 
The XRD results in Figure 4 represent the crystillanity index to the four PANI samples. The two broad peaks around $2 \theta=13^{\circ}$ and $25^{\circ}$ illustrates the PANI in amorphous status. The peak located at $2 \theta=25^{\circ}$ might be attributed to the polymer chain periodicity parallel, whereas the peaks around $2 \theta=13^{\circ}$ might be due to the polymer chain perpendicular periodicity [31]. There is no difference in XRD results while the change of the (dopant-monomer) ratios. However, the intensity of the peak at $\left(12^{\circ}\right.$ and $\left.25^{\circ}\right)$ slightly changed due to changing the ratio of concentration between the monomer and dopant. The diffraction peaks at $25^{\circ}$ accompanied with d-spacing ( $3.5 \AA$ ) indecates to the face-to-face interchain stacking distance between phenyl rings. Therefore, the peak intensity at $25^{\circ}$ increased simultaneously with improving $\pi-$ $\pi$ interchain of decreasing of background intensity. High planar chain conformation associated with the reduce the torsion angles among the benzen ring and backbone plane results extending in the activated conjugation length. The negligible low intensity of the peak at $25^{\circ}$ along with the increasing phosphoric acid concentrations, probably due to the structural deformation for the PANI chains [32].

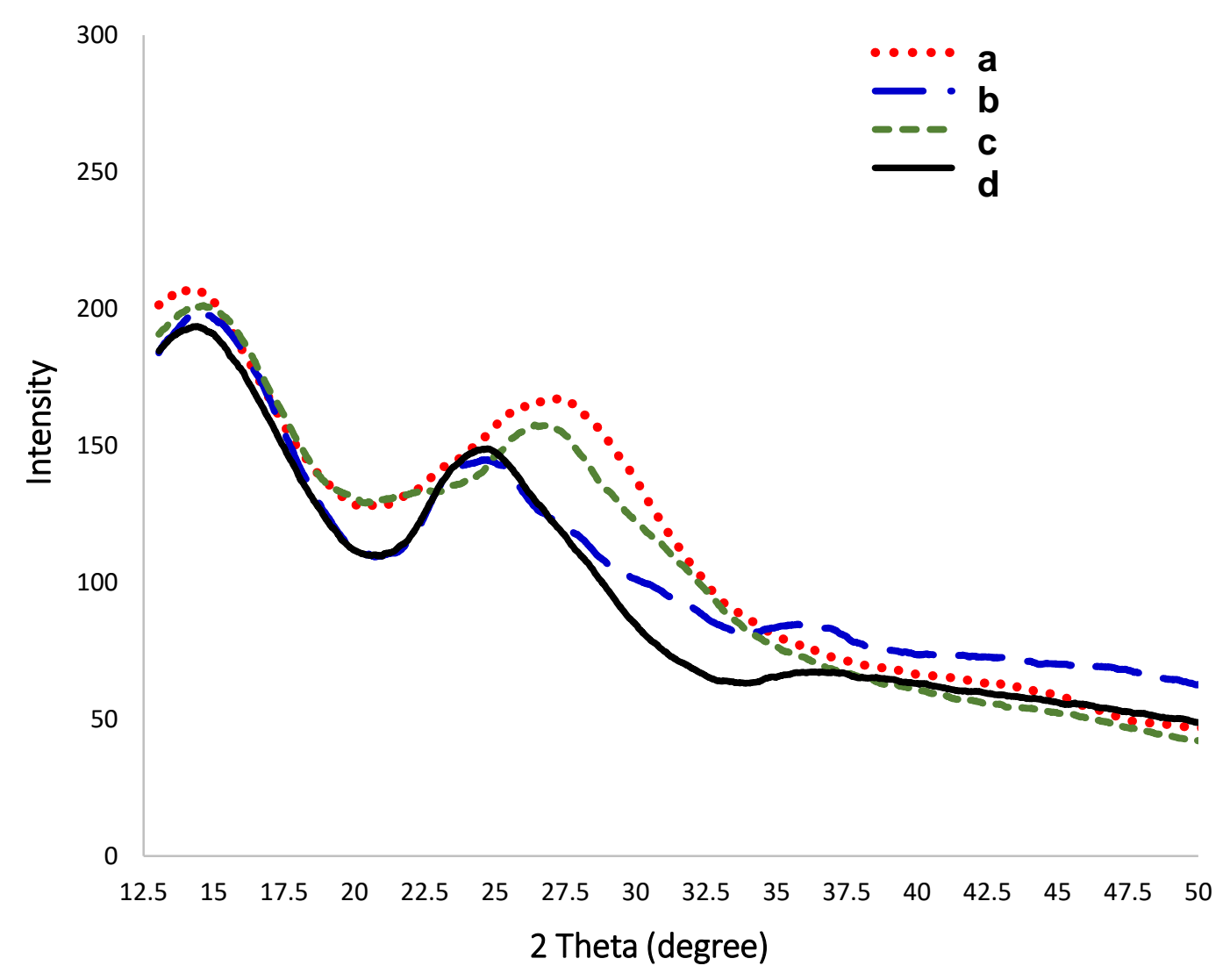

Figure 4. XRD for four PANI samples ;(a) nanofibers, (b) mix of nanospheres and nanofibers (c) nanorods and (d) nanospheres.

The electrical conductivity to the four PANI samples was measured using digital Multimeter through measuring the resistance at five different points and the average was taken then converted to conductivity using Equation 1.

$$
\sigma_{s}=\frac{1}{\rho_{s}}
$$


Where $\rho \mathbf{s}$ is surface resistivity in ohms/square and $\sigma \mathbf{s}$ is surface conductivity in Siemens/meter.

The PANI in powder form compressed into a pellet with $1.29 \mathrm{~cm}$ diameter and 0.19 $\mathrm{cm}$ thickness. The conductivities then measured at room temperature for the PANI in four different nanostructurs. The variation in the conductivity results often assigned to the differences in doping system and diversity in the PANI structures topology [21].

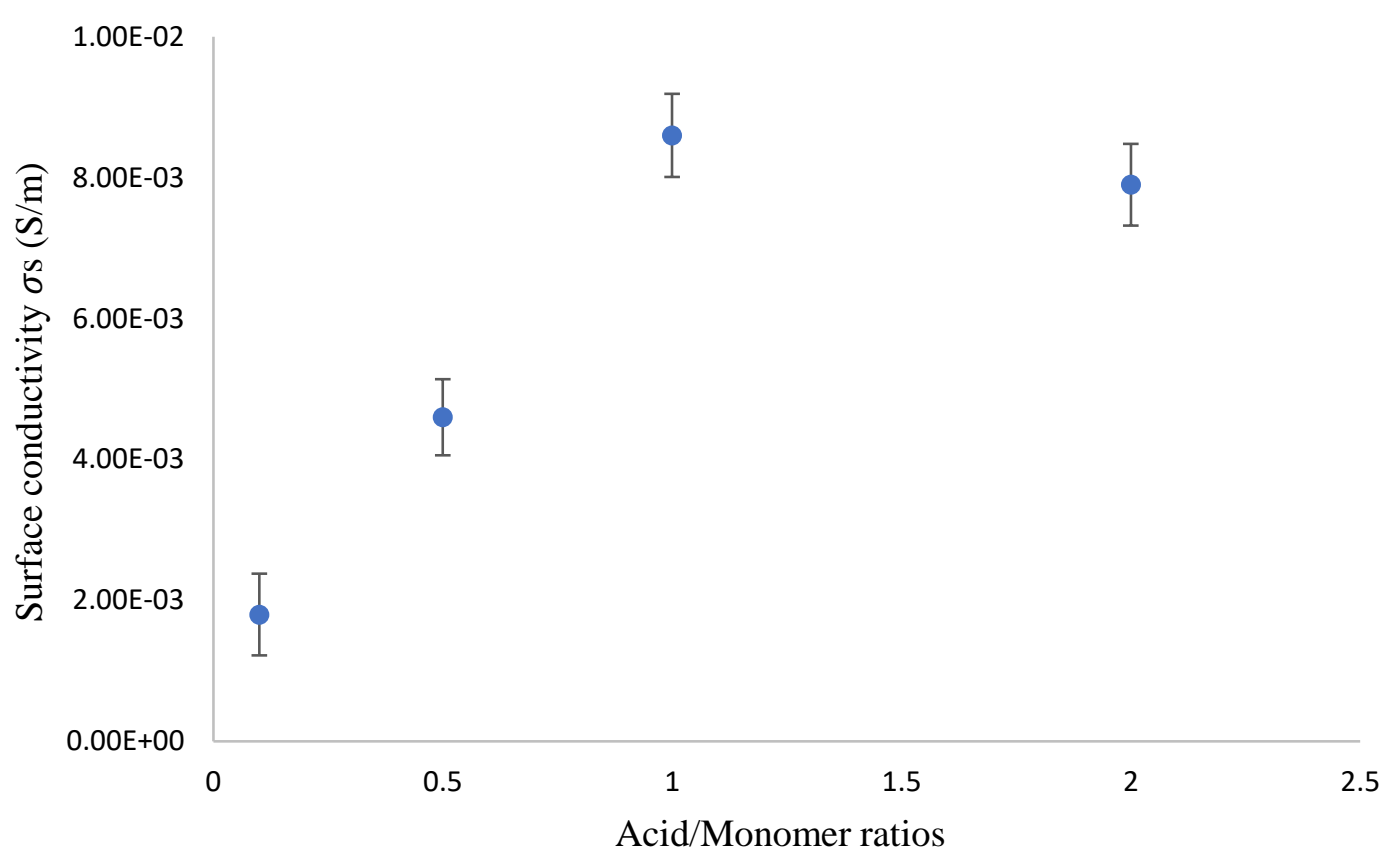

Figure 5. Conductivity measurments for PANI in various nanostructures

Despite to the acid type, the protonation level is highly elevate the electrical conductivity value in PANI. The interaction between PANI base and phosphoric acid proceed in the solid state, steady conductivity on composition of this mixtures are obtained. PANI protonation level base on the dilution of the protonic acid with good excessability of high acid content. However, Figure 5 shows the calculated molar fractions are plotted against of protonated PANI, the electrical conductivity dependence on degree of protonation. Commonly, the conductivity increases with more protonated unites in the polymer chain. Nevertheless, the nonuniform distribution of the PANI results lower percolation limit compared with high uniform distribution system of nanoparticles [33].

The results in Figure 5 suggests that the dopant ratios of PANI, main reason and basic roles of electon delever is based on doped chain in the PANI backbone. There is no constant proportional equation between dopant-monomer ratio and conductivity. However, the conductivity increases with increasing the dopend ratios until reach to equalize in the molar between monomer and dopant then the conductivity continue almost consistently.

The dispersion stability for PANI that illustrated in Figure 6 with four different nanostructures with the increasing the stability time. Once the dispersion of PANI in ethanol mediom by ultrasonication agitation stopped, the testing of dispersion stability was started by stopping the stirring action until 24 hours.

Conductive polymers in one dimensional nanostructures such as (fibers and rods) shows significant results for longer terms stability $[34,35]$. PANI nanofiber has the best stability in 
ethanol medium followed by PANI in nanoroad form. PANI sample consisting nanofiber nanosphere shapes has a third level of dispersion stability. However nanosphere showing the poorest stability properties among other nanostructures.

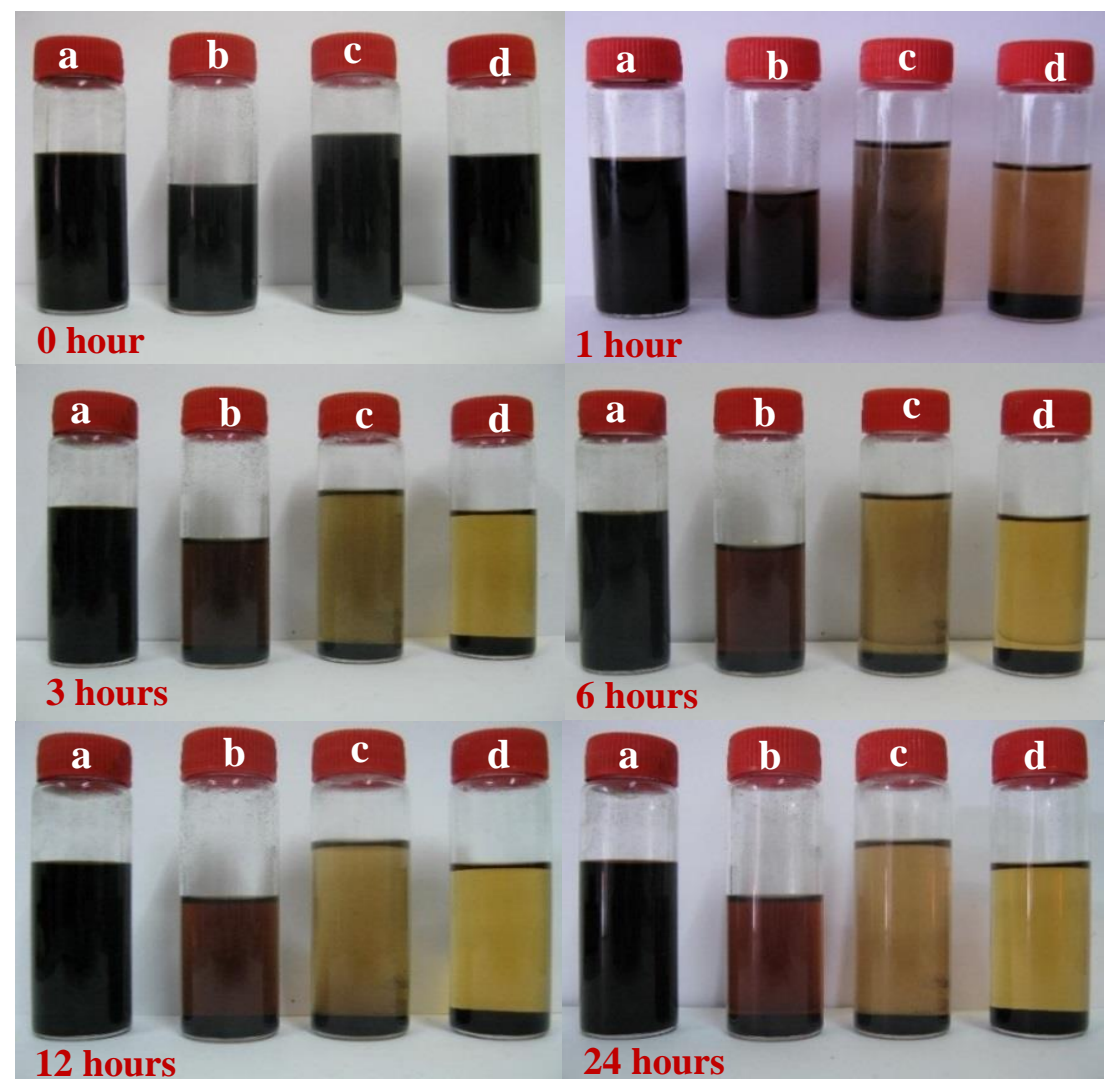

Figure 6. Dispersion stability of PANI; (a) nanofibers, (b) nanoroads, (c) mixture of nanofiber and nanosphers, (d) nanospheres

Nanofibers remained stable along the test but nanorod start partly sedimenting after six hours from the beginning of test. The PANI nanofibers has the least diameters among the samples. In other words, PANI in nanofibers obtained high surface area assist for steric stabilization attractions with solvent medium [36]. Salt spray and adhesion tests were evaluated after the 35 days of exposing samples in salt fog cabinet based on ASTM B117 standard. Adhesion test was applied based on standard ASTM D3359. Method B relied to evaluate coating performance, which is $5 \mathrm{~B}$ assign to the highest value and $0 \mathrm{~B}$ represent lowest value. Measuring coating thickness carried out based on ASTM D1186 standard and the average recorded in Table 2.

Table 2. Average coating thickness and adhesion strength.

\begin{tabular}{cccc}
\hline PANI Sample & $\begin{array}{c}\text { Lower thikness } \\
\text { coating value }\end{array}$ & $\begin{array}{c}\text { higer thikness } \\
\text { coating value }\end{array}$ & $\begin{array}{c}\text { Average of } \\
\text { adhesion test }\end{array}$ \\
\hline Nanofibers & 238 & 292 & $5 \mathrm{~B}$ \\
Nanorods & 234 & 293 & $5 \mathrm{~B}$ \\
Nanospheres & 246 & 339 & $3 \mathrm{~B}$ \\
\hline
\end{tabular}


After exposure to salt spray fog, Figure 7 have shown that the sample coated by nanospheres coating has too much exposing to corrosion through the big red rust spot of $\mathrm{Fe}_{3} \mathrm{O}_{4}$. The corrosion protection of conductive PANI consisting the redox process and uniform distribution of PANI in the coating containing. Which is assigned to the passive layer covered the sample and uniform dispersed of PANI nanoparticles that providing excelant barrier action [37]. The reduction of PANI (Emeraldine salt) status to PANI (Leucosalt) status accompanied with the release of phosphate dopant, prevent steel from corrosion aggression. The formation of passive film on iron samples assisted by phosphate ions at the defect spots. Leucosalt oxidation status is supposed belongs to re-oxidation subsequently by uptaking oxygen by the status of emeraldine salt [38].
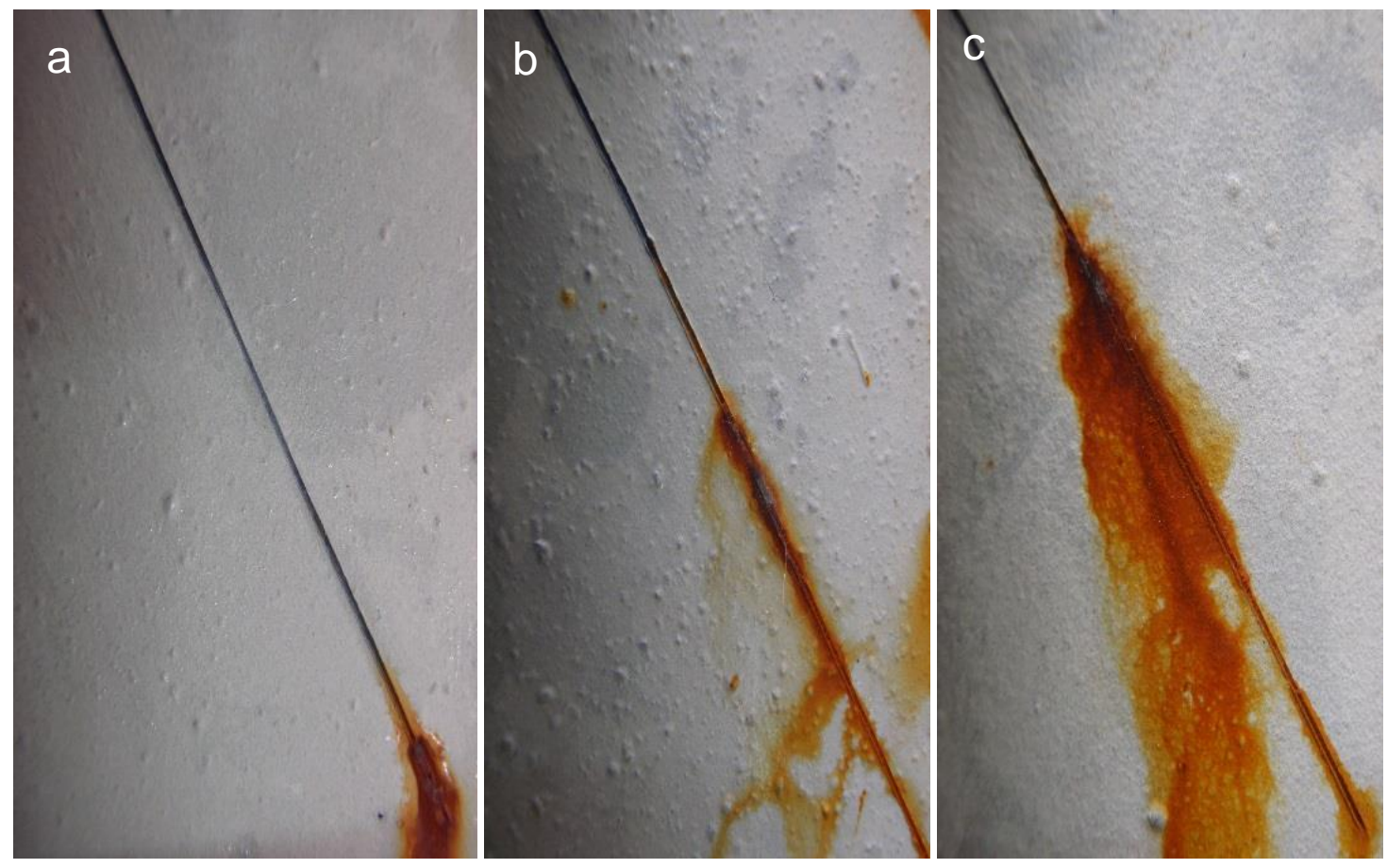

Figure 7. Carbon steel samples after 35 days exposed in salt spray chamber (a) nanofibers, (b) nanoroads, (c) nanspheres.

A function of the redox potential to form of diverse types of oxide preferred to form low potential samples. When high oxygen concentration at the metal surface, the formation of $\mathrm{Fe}_{3} \mathrm{O}_{4}$ occurs (Equation $4-6$ ). Due to the quantity of PANI and good distribution of nanofibers in the paint, only some of the samples suffered from $\mathrm{Fe}_{3} \mathrm{O}_{4}$. The better spread is the more compact to inhibit passing oxygen over the oxide, lead to the buildup the $\mathrm{Fe}_{3} \mathrm{O}_{4}$ layer [39].

$$
\begin{aligned}
& \mathrm{Fe}_{0}+\mathrm{H}_{2} \mathrm{O}=(\mathrm{FeOH})_{\mathrm{ads}}+\mathrm{H}^{+}+\mathrm{e}^{-} \\
& (\mathrm{FeOH})_{\mathrm{ads}}+\mathrm{H}_{2} \mathrm{O}=\left[(\mathrm{FeOH})_{2}\right]_{\mathrm{ads}}+\mathrm{H}^{+}+\mathrm{e}^{-} \\
& {\left[(\mathrm{FeOH})_{2}\right]_{\mathrm{ads}}+\mathrm{H}_{2} \mathrm{O}=\mathrm{FeOH}_{3}+\mathrm{H}^{+}+\mathrm{e}^{-}} \\
& 2 \mathrm{FeOH} 3=\mathrm{Fe}_{2} \mathrm{OH}_{3}+3 \mathrm{H}_{2} \mathrm{O} \\
& {\left[(\mathrm{FeOH})_{2}\right]_{\mathrm{ads}}+\mathrm{Fe}_{2} \mathrm{OH}_{3}=\mathrm{Fe}_{2} \mathrm{OH}_{4}+\mathrm{H}_{2} \mathrm{O}}
\end{aligned}
$$


The adhesion test was measured through cracking seven vertical lines crossed over seven horizontal lines. Thirty six squares resulted the crossed lines in one coating sample. Proper pull-up motion with special tape applied on these squares.

Results shows that coating containing PANI in nanofibers and nanoroads had the highest value of adhesion (5B). However, none of the squares is detached from metal plate. On the other hand, coating modified with PANI in nanospheres particles results 5 to $15 \%$ affected area (3B) after adhesion test. The authors attributed these results to the rust layer in-between carbon steel plate and coatings which is lowers the adhesion values. Accordingly, adhesion results indicate PANI nanofibers and nanorods provide way more corrosion protection than nanospheres due to the high rusting defects and delamination aspect.

\section{CONCLUSION}

PANI nanostructures were synthesized by in-situ polymerization method and blend with the acrylic paint then applied as anti-corrosion objects on the carbon steel panels. Four different nanostructures of PANI (nanofibers to nanorods and nanospheres) were tested for the behaviours of corrosion inhibitor. Corrosion values were evaluated visually by a digital camera and through using adhesion test. Results revealed that coating system with PANI nanofibers has the highest value of corrosion resistance due to the high dispersion stability and decent uniformity. However, this work finds that corrosion inhibition performance of PANI does not related with conductivity and doping values. Structure morphology and dispersion stability have a significant impact on the anticorrosion performance.

\section{ACKNOWLEDGEMENTS}

The authors would like to appreciation Research Management Centre at Universiti Teknologi Malaysia for managing financial support. The fundamental research grant Vot No. 77511 provided from Ministry of Higher Education (MOHE) is gratefully acknowledged. Sincere appreciation to the technical support of Polymer Laboratories and STATUS HEAD company at Johor state for the contribution in the adhesion and thickness tests.

\section{REFERENCES}

[1] Suriani M, Nik W, Mansor F, Jarkoni M, Maizurah C, Izwani I, Corrosion behavior and resistance parameters of silicon carbide nanocomposite coating on different metals. Journal of Mechanical Engineering and Sciences. 2018; 12: 3288-3301.

[2] Nik WW, Hajar H, Suriani M, Sabri M, Ghazali M, Development of anti-corrosive paint incorporated with henna extract as a natural inhibitor. Journal of Mechanical Engineering and Sciences. 2017; 11: 3179-3188.

[3] Hajar H, Ismail N, Zulkifli F, Sabri M, Nik W, Non-destructive tests on eco-friendly anti-corrosion paint. Journal of Mechanical Engineering and Sciences. 2017; 11: 28252833.

[4] Bhadra S, Khastgir D, Singha NK, Lee JH, Progress in preparation, processing and applications of polyaniline. Progress in Polymer Science. 2009; 34: 783-810.

[5] Kang E, Neoh K, Tan K, Polyaniline: a polymer with many interesting intrinsic redox states. Progress in Polymer Science. 1998; 23: 277-324.

[6] Deshpande PP, Jadhav NG, Gelling VJ, Sazou D, Conducting polymers for corrosion protection: a review. Journal of Coatings Technology and Research. 2014; 11: 473494. 
[7] Li Y, Zhang H, Wang X, Li J, Wang F, Growth kinetics of oxide films at the polyaniline/mild steel interface. Corrosion science. 2011; 53: 4044-4049.

[8] Al-Dulaimi A, Hashim S, Improving The Anti-Corrosion Properties Via Surface Modification For Silicon Dioxide By Conductive Polymer. International Journal Of Mechanical And Materials Engineering (IJMME). 2012; 7: 113-118.

[9] Tian Z, Yu H, Wang L, Saleem M, Ren F, Ren P, Chen Y, Sun R, Sun Y, Huang L, Recent progress in the preparation of polyaniline nanostructures and their applications in anticorrosive coatings. RSC Advances. 2014; 4: 28195-28208.

[10] Shi Y, Peng L, Ding Y, Zhao Y, Yu G, Nanostructured conductive polymers for advanced energy storage. Chemical Society Reviews. 2015; 44: 6684-6696.

[11] Guo B, Glavas L, Albertsson A-C, Biodegradable and electrically conducting polymers for biomedical applications. Progress in Polymer Science. 2013; 38: 1263 1286.

[12] Zhang Z, Wei Z, Zhang L, Wan M, Polyaniline nanotubes and their dendrites doped with different naphthalene sulfonic acids. Acta Materialia. 2005; 53: 1373-1379.

[13] Fu G, Zhao J, Sun Y, Kang E, Neoh K, Conductive hollow nanospheres of polyaniline via surface-initiated atom transfer radical polymerization of 4-vinylaniline and oxidative graft copolymerization of aniline. Macromolecules. 2007; 40: 2271-2275.

[14] Virji S, Huang J, Kaner RB, Weiller BH, Polyaniline nanofiber gas sensors: examination of response mechanisms. Nano letters. 2004; 4: 491-496.

[15] Long Y-Z, Li M-M, Gu C, Wan M, Duvail J-L, Liu Z, Fan Z, Recent advances in synthesis, physical properties and applications of conducting polymer nanotubes and nanofibers. Progress in Polymer Science. 2011; 36: 1415-1442.

[16] Chiou N-R, Lee LJ, Epstein AJ, Self-assembled polyaniline nanofibers/nanotubes. Chemistry of materials. 2007; 19: 3589-3591.

[17] Ding X, Han D, Wang Z, Xu X, Niu L, Zhang Q, Micelle-assisted synthesis of polyaniline/magnetite nanorods by in situ self-assembly process. Journal of Colloid and Interface science. 2008; 320: 341-345.

[18] Xia H, Narayanan J, Cheng D, Xiao C, Liu X, Chan HSO, Formation of ordered arrays of oriented polyaniline nanoparticle nanorods. The Journal of Physical Chemistry B. 2005; 109: 12677-12684.

[19] Zhang D, Wang Y, Synthesis and applications of one-dimensional nano-structured polyaniline: An overview. Materials Science and Engineering: B. 2006; 134: 9-19.

[20] Zhang Z, Wei Z, Wan M, Nanostructures of polyaniline doped with inorganic acids. Macromolecules. 2002; 35: 5937-5942.

[21] Amarnath CA, Kim J, Kim K, Choi J, Sohn D, Nanoflakes to nanorods and nanospheres transition of selenious acid doped polyaniline. Polymer. 2008; 49: 432437.

[22] Tran HD, D'Arcy JM, Wang Y, Beltramo PJ, Strong VA, Kaner RB, The oxidation of aniline to produce "polyaniline": a process yielding many different nanoscale structures. Journal of Materials Chemistry. 2011; 21: 3534-3550.

[23] Sathiyanarayanan S, Syed Azim S, Venkatachari G, Performance studies of phosphate-doped polyaniline containing paint coating for corrosion protection of aluminium alloy. Journal of Applied Polymer Science. 2008; 107: 2224-2230.

[24] Kalendová A, Veselý D, Stejskal J, Trchová M, Anticorrosion properties of inorganic pigments surface-modified with a polyaniline phosphate layer. Progress in Organic Coatings. 2008; 63: 209-221.

[25] Al-Dulaimi AA, Hashim S, Khan M, Corrosion protection of carbon steel using polyaniline composite with inorganic pigments. Sains Malaysiana. 2011; 40: 757-763. 
[26] Vittal R, Gomathi H, Kim K-J, Beneficial role of surfactants in electrochemistry and in the modification of electrodes. Advances in colloid and interface science. 2006; 119: 55-68.

[27] Li G, Pang S, Liu J, Wang Z, Zhang Z, Synthesis of polyaniline submicrometer-sized tubes with controllable morphology. Journal of Nanoparticle Research. 2006; 8: 10391044.

[28] Zhang L, Zhang L, Wan M, Wei Y, Polyaniline micro/nanofibers doped with saturation fatty acids. Synthetic metals. 2006; 156: 454-458.

[29] Cao Y, Li S, Xue Z, Guo D, Spectroscopic and electrical characterization of some aniline oligomers and polyaniline. Synthetic metals. 1986; 16: 305-315.

[30] Tang J, Jing X, Wang B, Wang F, Infrared spectra of soluble polyaniline. Synthetic metals. 1988; 24: 231-238.

[31] Pouget J, Jozefowicz M, Epstein Aea, Tang X, MacDiarmid A, X-ray structure of polyaniline. Macromolecules. 1991; 24: 779-789.

[32] Rana U, Chakrabarti K, Malik S, Benzene tetracarboxylic acid doped polyaniline nanostructures: morphological, spectroscopic and electrical characterization. Journal of Materials Chemistry. 2012; 22: 15665-15671.

[33] Stejskal J, Sapurina I, Trchová M, Prokeš J, Křivka I, Tobolková E, Solid-state protonation and electrical conductivity of polyaniline. Macromolecules. 1998; 31: 2218-2222.

[34] Al-Dulaimi AA, Wan Rosli W, Water Dispersion Conductive Polypyrrole Based on Nanocrystalline Cellulose. Advanced Materials Research. 2014; 1043: 105-108.

[35] Al-Dulaimi AA, Wanrosli W, Preparation of Colloidal Properties and Water Dispersible Conductive Polypyrrole Nanocomposite of Nanocrystalline Cellulose. Polymers \& Polymer Composites. 2016; 24: 695.

[36] Li X, Zhuang T, Wang G, Zhao Y, Stabilizer-free conducting polyaniline nanofiber aqueous colloids and their stability. Materials Letters. 2008; 62: 1431-1434.

[37] Chen F, Liu P, Conducting polyaniline nanoparticles and their dispersion for waterborne corrosion protection coatings. ACS applied materials \& interfaces. 2011; 3: 2694-2702.

[38] Sathiyanarayanan S, Azim SS, Venkatachari G, Preparation of polyaniline-TiO2 composite and its comparative corrosion protection performance with polyaniline. Synthetic metals. 2007; 157: 205-213.

[39] Gonçalves G, Baldissera A, Rodrigues Jr L, Martini E, Ferreira C, Alkyd coatings containing polyanilines for corrosion protection of mild steel. Synthetic metals. 2011; 161: 313-323. 